\title{
Effect of acute resistance exercise on carotid artery stiffness and cerebral blood flow pulsatility
}

\author{
Wesley K. Lefferts, Jacqueline A. Augustine and Kevin S. Heffernan*
}

Department of Exercise Science, Syracuse University, Syracuse, NY, USA

\section{Edited by:}

Phil Neil Ainslie, University of British Columbia, Canada

Reviewed by:

Samuel J.E. Lucas, University of Birmingham, UK

Aaron Alexander Phillips, University of British Columbia, Canada

*Correspondence:

Kevin S. Heffernan, The Human

Performance Laboratory,

Department of Exercise Science,

Syracuse University, 820 Comstock

Ave., Syracuse, NY 13244, USA

e-mail:ksheffer@syr.edu
Arterial stiffness is associated with cerebral flow pulsatility. Arterial stiffness increases following acute resistance exercise (RE). Whether this acute RE-induced vascular stiffening affects cerebral pulsatility remains unknown. Purpose: To investigate the effects of acute RE on common carotid artery (CCA) stiffness and cerebral blood flow velocity (CBFv) pulsatility. Methods: Eighteen healthy men (22 $\pm 1 \mathrm{yr} ; 23.7 \pm 0.5 \mathrm{~kg} \cdot \mathrm{m}^{-2}$ ) underwent acute RE (5 sets, 5-RM bench press, 5 sets 10-RM bicep curls with $90 \mathrm{~s}$ rest intervals) or a time control condition (seated rest) in a randomized order. CCA stiffness ( $\beta$-stiffness, Elastic Modulus (Ep)) and hemodynamics (pulsatility index, forward wave intensity, and reflected wave intensity) were assessed using a combination of Doppler ultrasound, wave intensity analysis and applanation tonometry at baseline and 3 times post-RE. CBFv pulsatility index was measured with transcranial Doppler at the middle cerebral artery (MCA). Results: CCA $\beta$-stiffness, Ep and CCA pulse pressure significantly increased post-RE and remained elevated throughout post-testing $(p<0.05)$. No changes in MCA or CCA pulsatility index were observed $(p>0.05)$. There were significant increases in forward wave intensity post-RE $(p<0.05)$ but not reflected wave intensity $(p>0.05)$. Conclusion: Although acute RE increases CCA stiffness and pressure pulsatility, it does not affect CCA or MCA flow pulsatility. Increases in pressure pulsatility may be due to increased forward wave intensity and not pressure from wave reflections.

Keywords: arterial stiffness, blood pressure, exercise, wave reflection

\section{INTRODUCTION}

Increases in arterial stiffness with age and/or disease increases risk for cardiovascular events such as myocardial infarction and stroke (Sutton-Tyrrell et al., 2005; Mitchell et al., 2010). Increased arterial stiffness also contributes to target organ damage such as renal dysfunction and retinal damage (Katsi et al., 2012; Safar et al., 2012). The elastic properties of the large central arteries (i.e., aorta and carotid) function to dampen the amplitude of fluctuations in pressure and flow, thereby preventing transmission of excess energy into target organs (Mitchell et al., 2011). Similar to the kidney and eye, the brain is a high flow organ particularly susciptible to hemodynamic pulsatility (Mitchell, 2008). Repeated exposure of the cerebral vasculature to pulsatile pressure/flow may precipitate microvascular hypoperfusion and subsequent ischemia contributing to rarefaction, white matter hyperintensities and ultimately cerebrovascular impairment (Mitchell et al., 2005). Recent studies note a strong assocation between arterial stiffness, pressure/flow pulsatility and cerebral perfusion (Kwater et al.,

\footnotetext{
Abbreviations: RE, Resistance exercise; CBFv, Cerebral blood flow velocity; RM, Repetition maximum; SBP, Systolic blood pressure; DBP, Diastolic blood pressure; CCA, Common carotid artery; IMT, Intima-media thickness; MAP, Mean arterial pressure; PI, Pulsatility index; WIA, Wave intensity analysis; NA, Negative area; Ep, Elastic modulus; $\beta, \beta$ Stiffness index, PP, Pulse pressure; Aix, Augmentation index; WSA, Wave separation analysis; Pf, Forward wave pressure; Pb, Backwards wave pressure; MCA, Middle cerebral artery; TCD, Transcranial Doppler; CVRi, Cerebrovascular resistance index.
}

2009; Tarumi et al., 2011; Webb et al., 2012). Studies to date examining associations between arterial stiffness and cerebrovascular pulsatility have been cross-sectional. It is largely unknown if acute elevations in arterial stiffness affect cerebrovascular pulsatility.

Resistance exercise (RE) is an accepted lifestyle strategy to increase muscular strength and attenuate sarcopenia-related functional impairment (Miyachi et al., 2004; Miyachi, 2013) and is currently recommended by major health organizations for health promotion/disease prevention (Pollock et al., 2000). Paradoxically, despite functional and musculoskeletal benefits, RE may impair vascular function (Miyachi et al., 2004; Heffernan et al., 2007b; Collier et al., 2010). Higher intensity RE training typically increases arterial stiffness (Miyachi, 2013) and recent evidence suggests that RE training may contribute to reductions in cerebral blood flow velocity (CBFv) (Jung et al., 2012). Acute $\mathrm{RE}$ has consistently been shown to increase large central artery stiffness (Heffernan et al., 2007a; Collier et al., 2010) immediately after and for upwards of $30 \mathrm{~min}$ after perturbation (DeVan et al., 2005). Given the strong cross-sectional association between arterial stiffness, pressure/flow pulsatility, and cerebral perfusion, it is possible that acute RE-induced increases in arterial stiffness may transiently increase $\mathrm{CBFv}$ pulsatility. The purpose of this study was to test the hypothesis that acute RE-induced increases in arterial stiffness would lead to increases in carotid pressure/flow pulsatility and $\mathrm{CBFv}$ pulsatility. 


\section{METHODS \\ PARTICIPANTS}

Eighteen recreationally active men, ranging in age from 19-28 yrs, were recruited from the local University community for this randomized, crossover-design study. None were highly trained in an exclusive exercise modality (i.e., distance runners, bodybuilders, or power lifters). Exclusion criteria included self-reported smoking, hypertension, diabetes mellitus, hyperlipidemia, pulmonary disease, renal disease, neurological disease or peripheral artery disease and/or use of medications of any kind. This study was approved by the Institutional Review Board of Syracuse University and all participants provided written informed consent prior to study initiation. Vascular testing was conducted at the same time of day in a quiet, dimly lit, temperature-controlled laboratory. Participants were instructed to fast for $\geq 3 \mathrm{~h}$ and avoid vigorous exercise and consuming caffeine/alcohol $\geq 12 \mathrm{~h}$ before testing. Height and weight were assessed via wall-mounted ruler and electronic scale, respectively, and body composition was estimated via air displacement plethysmography (BodPod; COSMED, Concord, CA).

\section{DESIGN}

Upon arrival, participants rested for $10 \mathrm{~min}$ in the supine position. This was followed by all vascular and hemodynamic measures. Participants then performed (a) upper body RE, or (b) seated rest with minimal movement (time control) in a randomized order on two separate days. The RE protocol consisted of 5 sets of 5-repetitions for bench press and 5 sets of 10-repetitions for biceps curl, with each set being separated by $90 \mathrm{~s}$ of rest. Exercises were performed at $100 \%$ of participants 5 repetition maximum (RM) and $10 \mathrm{RM}$ for bench press and biceps curl respectively. If participants could not complete the designated number of repetitions, a "drop set" was instituted whereby the total load was reduced in small increments until participants successfully completed target repetitions. Participants RMs for each exercise were determined previously on a separate day. This acute RE protocol has previously been shown to acutely increase arterial stiffness (Fahs et al., 2009). Post-testing vascular and hemodynamic measures were made 10, 20, and 30 min following acute RE based on previous findings noting prominent changes in arterial stiffness to occur at these times (DeVan et al., 2005; Heffernan et al., 2007a; Fahs et al., 2009). Carotid pressure, flow, stiffness, and cerebral velocity measures were obtained simultaneously.

\section{MEASURES}

\section{Brachial blood pressure}

Systolic blood pressure (SBP) and diastolic brachial blood pressure (DBP) was measured prior to each set of vascular measures (baseline, post-1, post-2, post-3) using a validated, automated oscillometric cuff (EW3109, Panasonic Electric Works, Secaucus NJ). Pressures were taken in duplicate and averaged. If values were different by more than $5 \mathrm{mmHg}$ a third measure was obtained and the average of the 2 closest measures used for subsequent analyses.

\section{Carotid doppler ultrasonography}

Images of the common carotid artery (CCA) were obtained using Doppler ultrasound (ProSound $\alpha 7$, Aloka, Tokyo, Japan) and
7.5-10.0 $\mathrm{mHz}$ linear-array probe. CCA intima-media thickness (IMT) was assessed using a longitudinal view with both near wall and far wall lumen-IMT boundaries visible. Wall thickness was measured across a $5 \mathrm{~mm}$ region of interest via semi-automated digital calipers during systole and diastole (determined from ECG gating). The distance from the lumen-intima interface to the media-adventitia interface was measured as the IMT. CCA systolic and diastolic diameters were measured from inside the near-wall IMT to far-wall IMT. CCA mean blood velocities $(V m)$ were measured using Doppler-ultrasound and calculated as: $V m=\int V(t) d t / F T$, where $\int V(t) d t$ is the velocity-time integral of the velocity waveform and FT is flow time. CCA flow and shear rate were calculated as $\pi \times(1 / 3$ systolic radius + $2 / 3$ diastolic radius $)^{2} \times V m \times 60$ and $4 \times(\mathrm{Vm} /$ systolic diameter), respectively. CCA mean circumferential wall tension was calculated as [mean arterial pressure $\mathrm{x}$ mean radius], where mean arterial pressure (MAP) is expressed in dyne $\cdot \mathrm{cm}^{2}$. CCA tensile wall stress was calculated by dividing circumferential wall tension by IMT where both mean radius and IMT are expressed in $\mathrm{cm}$ (Carallo et al., 1999) CCA pulsatility index (PI) was calculated with a semi-automated flow tracing software using the following equation: $\left(V_{s}-V_{d}\right) /$ mean $V$, where $V_{s}$ is the peak systolic velocity, $V_{d}$ diastolic velocity and mean $V$ the mean velocity. CCA flow resistance index (RI) was calculated as $\left(V_{s}-V_{d}\right) / V_{s}$.

Wave intensity analysis (WIA) combined with eTracking was used to derive forward and reflected wave intensity as measures related to pulsatile cerebrovascular burden and arterial stiffness in the carotid artery. WIA was performed simultaneously with carotid applanation tonometry on the contralateral artery. The distance from the near wall to far wall lumen-intima interface is continuously traced using eTracking software, creating a distension waveform almost identical to pressure waveforms (Van Bortel et al., 2001; Niki et al., 2002). WIA distension waveforms were calibrated against carotid systolic and diastolic pressures obtained via applanation tonometry described below. Flow waveforms were measured using range gated color Doppler signals averaged along the Doppler beam. An insonation angle $\leq 60^{\circ}$ was maintained for all measures and sample volume was manually adjusted to encompass the entire vessel. At least 8 carotid waveforms were averaged to gain a representative average waveform. Wave intensity was calculated using time derivatives of blood pressure $(\mathrm{P})$ and velocity $(\mathrm{U})$, where wave intensity $=(d P / d t \times d U / d t)$; the area under the $d P / d t \times d U / d t$ curve represents the energy transfer of the wave (Sugawara et al., 2009b). $\mathrm{W}_{1}$ represents a forward compression wave produced during early systole, accelerating flow and increasing pressure; 2 the negative area (NA) occurring immediately after $\mathrm{W}_{1}$ is a backward travelling compression wave due to reflected waves from the periphery that decelerate flow but increase pressure. NA measured in the CCA has been suggesting as a measure of cerebrovascular tone (Bleasdale et al., 2003).

Arterial stiffness measures included beta stiffness index $(\beta)$, and Peterson's pressure-strain elastic modulus (Ep) and were calculated as:

$$
\beta=\ln \left(\mathrm{P}_{\mathrm{Max}} / \mathrm{P}_{\mathrm{Min}}\right) /\left[\left(\mathrm{D}_{\mathrm{Max}}-\mathrm{D}_{\text {Min }}\right) / \mathrm{D}_{\text {Min }}\right]
$$




$$
\mathrm{Ep}=\left(\mathrm{P}_{\mathrm{Max}}-\mathrm{P}_{\text {Min }}\right) /\left[\left(\mathrm{D}_{\mathrm{Max}}-\mathrm{D}_{\mathrm{Min}}\right) / \mathrm{D}_{\mathrm{Min}}\right]
$$

where $\mathrm{P}$ and $\mathrm{D}$ correspond to pressure and diameter respectively, and Max and Min refer to maximum (systolic) and minimum (diastolic) values during the cardiac cycle. CCA characteristic impedance $(\mathrm{Zc})$ was calculated by re-arranging the WaterHammer equation as $Z c=(P W V x \rho) / A$, where $\rho$ is blood density (assumed constant $1.055 \mathrm{~kg} / \mathrm{cm}^{3}$ ) and A is carotid area. A onepoint pulse wave velocity (PWV) was derived from local wave speed $(c)$ as $(\beta P / 2 \rho)^{1 / 2}$ (Harada et al., 2002; Hanya, 2013).

\section{Carotid and aortic blood pressure waveform analysis}

Pressure waveforms were obtained simultaneously in the contralateral CCA from a $10 \mathrm{~s}$ epoch and measured in duplicate using applanation tonometry (SphygmoCor, AtCor Medical, Syndey, Australia). CCA pressure waveforms were calibrated to brachial MAP and DBP. Additionally, aortic pressure waveforms were derived from radial pressure waveforms measured in duplicate $(10 \mathrm{~s}$ epochs) and a generalized transfer function. Pulse pressure (PP) was calculated as SBP minus DBP. Augmentation index was calculated as the difference between the early- and late systolic peaks of the pressure waveforms to the total $\mathrm{PP}$ expressed as a percentage $(\mathrm{P} 2-\mathrm{P} 1 / \mathrm{PP} \times 100)$ and standardized to a heart rate of 75 beats per min (AIx75).

Wave separation analyses (WSA) were performed in order to obtain complimentary data to WIA and to gain further insight into origins of pressure pulsatility following acute RE. Pressure waveforms were separated into forward (Pf) and backwards/reflected $(\mathrm{Pb})$ components. This technique uses a modified average-flow waveform based on the original flow triangulation method of Westerhof et al. and has been described previously in detail (Westerhof et al., 2006). Additionally, aortic pulse wave velocity (PWV) was estimated using the time lag between aortic $\mathrm{Pf}$ and $\mathrm{Pb}$ (Qasem and Avolio, 2008). Carotid-femoral distance was estimated by multiplying body height by 0.29 which may reduce measurement bias due to body disproportion that can occur with the standard tape measure method (Filipovsky et al., 2010).

\section{Cerebral blood flow velocity}

Middle cerebral artery (MCA) blood velocity was assessed using a 2-mHz transcranial Doppler (TCD) ultrasound probe (DWL Doppler Box-X, Compumedics, Germany) applied to the temporal window. Mean $\mathrm{CBFv}$ and pulsatility index were measured at a depth of 50-65 mm (Jung et al., 2012; Xu et al., 2012). The envelope of the velocity spectrum and mean velocity were calculated by a standard algorithm implemented on the instrument with use of a fast Fourier transform. MCA PI was calculated via an automated waveform tracking function using the same equation described for CCA PI. Cerebrovascular resistance index (CVRi) was calculated as $\mathrm{MAP} / V_{\text {mean }}$.

\section{STATISTICAL ANALYSES}

All data is reported as mean \pm standard error of the mean and statistical significance was established a priori as $p<0.05$. Normality of distribution for variables was assessed qualitatively using histograms and Q-Q plots as well as quantitatively using the
Shapiro-Wilk test. Data that were not normally distributed were $\log$ transformed to meet assumptions for parametric analyses. An analysis of variance with repeated measures ( 2 conditions $x$ 4 time points) was used to analyze main outcome variables. Post-hoc $t$-tests were used to investigate significant interactions.

\section{RESULTS}

Participants' age, body mass index, and body fat were, $22 \pm$ 1 years, $23.7 \pm 0.5 \mathrm{~kg} \cdot \mathrm{m}^{-2}$ and $11.4 \pm 0.9 \%$, respectively. The average load for the 5-RM bench press and 10-RM biceps curl were $76 \pm 4$ and $32 \pm 2 \mathrm{~kg}$, respectively. Carotid and cerebral measures are displayed independently in Tables $\mathbf{1 - 5}$. There were significant group-by-time interactions for brachial and carotid SBP, DBP, and PP. Post-hoc analyses revealed brachial SBP significantly increased immediately following RE and remained elevated for all post-RE time points compared to baseline $(p<0.05$; Table 1). Similarly, carotid SBP at post-1 was significantly different from baseline following RE $(p<0.05$; Table 1). Carotid SBP at post-2 and post-3 did not differ from baseline for the RE condition $(p>0.05)$. DBP decreased post-exercise compared to baseline for all time points resulting in overall increases in PP $(p<0.05$; Table 1). There was a significant interaction detected for heart rate, as it was significantly elevated following RE for the duration of post-testing compared to baseline $(p<0.05$; Table 1$)$. Changes in carotid PP are displayed in Figure 1.

There were no significant changes in CCA systolic or diastolic IMT (data not shown), CCA mean blood flow, or CCA PI following acute RE (Table 2 and Figure 2). Both CCA Ep and $\beta$-stiffness significantly increased following RE, and remained significantly elevated throughout post-testing $(p<0.05$; Table 3$)$. Changes in $\beta$-stiffness are displayed in Figure 3. A condition-bytime interaction was detected for carotid $\mathrm{W}_{1}$, which significantly increased post-RE and remained elevated throughout post-testing ( $p<0.05$; Table 3). Carotid AIx75 was not significantly altered by acute RE $(p>0.05)$. NA was not normally distributed and was log transformed to meet assumptions for parametric statistical analyses. There were no statistically significant changes in carotid lnNA $(p>0.05)$. WSA could not be performed for 6 participants as reflection time was outside of the measurement device's acceptable range $(<50 \mathrm{~ms})$ for calculations. Data for the remaining 12 are reported herein. Carotid Pf had a significant condition-by-time interaction; post-hoc testing revealed Pf significantly increased following RE, remaining elevated through post-3 $(p<0.05$; Table 3$)$. There were no statistically significant changes in carotid $\mathrm{Pb}(p>0.05)$.

There was a significant interaction for aortic AIx75 $(p<0.05)$ and a trend for an interaction for aortic stiffness as values were elevated following acute RE but not following the time control $(p=0.08$; Table 4). There was no significant change in aortic Pf or $\mathrm{Pb}$ following acute $\mathrm{RE}(p>0.05)$. A significant condition-bytime interaction was detected for aortic time to $\mathrm{Pb}$, indicative of faster wave reflection speed. Values significantly decreased post$\mathrm{RE}$ compared to baseline and remained different through post$3(p<0.05)$. Mean MCA CBFv, MCA PI (Figure 4), and MCA CVRi did not differ across time or between conditions $(p>0.05$, Table 5). 
Table 1 | Common carotid artery (CCA) and brachial pressures across testing time points between control and resistance exercise $(n=18)$.

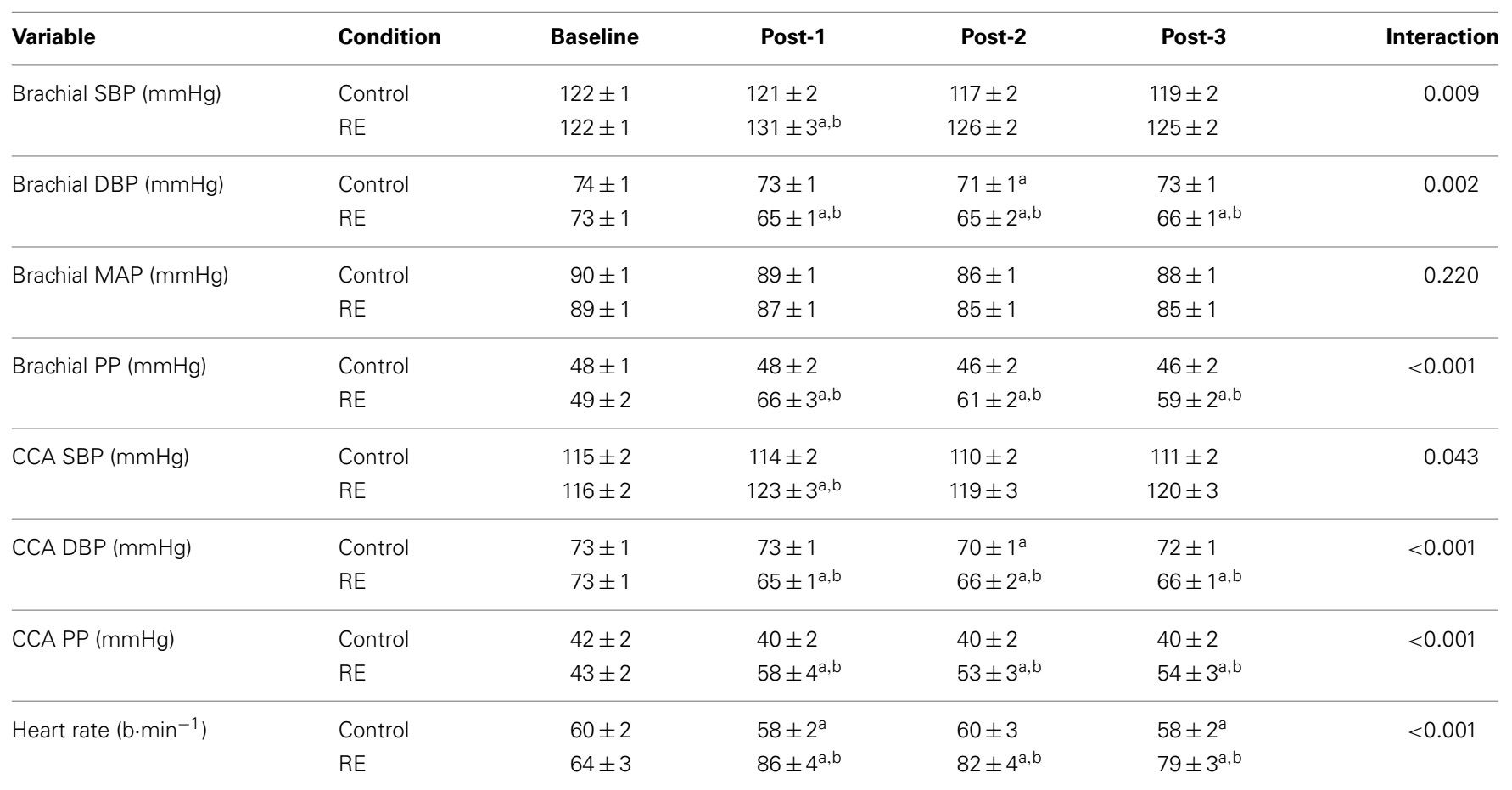

a Significantly different from within-condition baseline, $p<0.05$.

b Significantly different from other condition, same time point, $p<0.05$.

Table 2 | Common carotid dimensions/hemodynamics across testing time points between control and resistance exercise ( $n=18$ ).

\begin{tabular}{|c|c|c|c|c|c|c|}
\hline Variable & Condition & Baseline & Post-1 & Post-2 & Post-3 & Interaction \\
\hline \multirow[t]{2}{*}{ Mean diameter (mm) } & Control & $5.54 \pm 0.09$ & $5.55 \pm 0.11$ & $5.57 \pm 0.10$ & $5.69 \pm 0.10$ & 0.539 \\
\hline & $\mathrm{RE}$ & $5.48 \pm 0.11$ & $5.35 \pm 0.11$ & $5.51 \pm 0.11$ & $5.54 \pm 0.09$ & \\
\hline \multirow[t]{2}{*}{ Blood flow $\left(\mathrm{mL} \cdot \mathrm{s}^{-1}\right)$} & Control & $612.5 \pm 21.3$ & $656.6 \pm 36.4$ & $616.7 \pm 20.8$ & $626.3 \pm 29.7$ & 0.255 \\
\hline & $\mathrm{RE}$ & $635.0 \pm 27.6$ & $664.8 \pm 35.4$ & $700.3 \pm 28.8$ & $672.5 \pm 26.7$ & \\
\hline \multirow[t]{2}{*}{ Shear rate $\left(\mathrm{sec}^{-1}\right)$} & Control & $279.1 \pm 13.1$ & $293.7 \pm 17.5$ & $275.9 \pm 14.2$ & $259.3 \pm 9.6$ & 0.105 \\
\hline & $\mathrm{RE}$ & $296.9 \pm 12.7$ & $339.9 \pm 16.7$ & $336.2 \pm 15.9$ & $305.76 \pm 10.9$ & \\
\hline \multirow[t]{2}{*}{ Mean circumferential wall tension (dynes $\cdot \mathrm{cm}^{-1}$ ) } & Control & $3.4 \pm 0.1$ & $3.4 \pm 0.1$ & $3.3 \pm 0.1$ & $3.4 \pm 0.1$ & 0.287 \\
\hline & $\mathrm{RE}$ & $3.3 \pm 0.1$ & $3.2 \pm 0.1$ & $3.2 \pm 0.1$ & $3.2 \pm 0.1$ & \\
\hline \multirow[t]{2}{*}{ Mean tensile stress $\left(10^{4}\right.$ dynes $\left.\cdot \mathrm{cm}^{-1}\right)$} & Control & $89.5 \pm 2.9$ & $92.2 \pm 2.7$ & $84.0 \pm 2.9$ & $91.4 \pm 3.8$ & 0.157 \\
\hline & $\mathrm{RE}$ & $87.5 \pm 3.2$ & $81.2 \pm 3.8$ & $78.9 \pm 3.1$ & $81.5 \pm 3.2$ & \\
\hline \multirow[t]{2}{*}{ Resistance Index } & Control & $0.77 \pm 0.01$ & $0.77 \pm 0.01$ & $0.76 \pm 0.01$ & $0.76 \pm 0.01$ & 0.013 \\
\hline & $\mathrm{RE}$ & $0.76 \pm 0.01$ & $0.80 \pm 0.01^{a, b}$ & $0.78 \pm 0.01$ & $0.78 \pm 0.01$ & \\
\hline \multirow[t]{2}{*}{ Pulsatility Index } & Control & $2.07 \pm 0.08$ & $2.10 \pm 0.08$ & $2.06 \pm 0.10$ & $2.11 \pm 0.09$ & 0.094 \\
\hline & $\mathrm{RE}$ & $2.01 \pm 0.09$ & $2.16 \pm 0.08$ & $1.95 \pm 0.05$ & $2.02 \pm 0.06$ & \\
\hline
\end{tabular}

a Significantly different from within-condition Baseline, $p<0.05$.

${ }^{b}$ Significantly different from other condition, same time point, $p<0.05$.

\section{DISCUSSION}

The novel finding of the present study was that while acute RE does increase carotid artery stiffness and pressure pulsatility, acute RE may not affect carotid or cerebral flow pulsatility.
Moreover, using novel methods of hemodynamic appraisal (WIA and WSA) we provide unique insight into the origin of increased pressure pulsatility after acute RE; increased pressure pulsatility in the carotid artery is likely due to increased forward wave 
Table 3 | Measures of common carotid wave reflection/stiffness across testing time points between control and resistance exercise $(n=18)$.

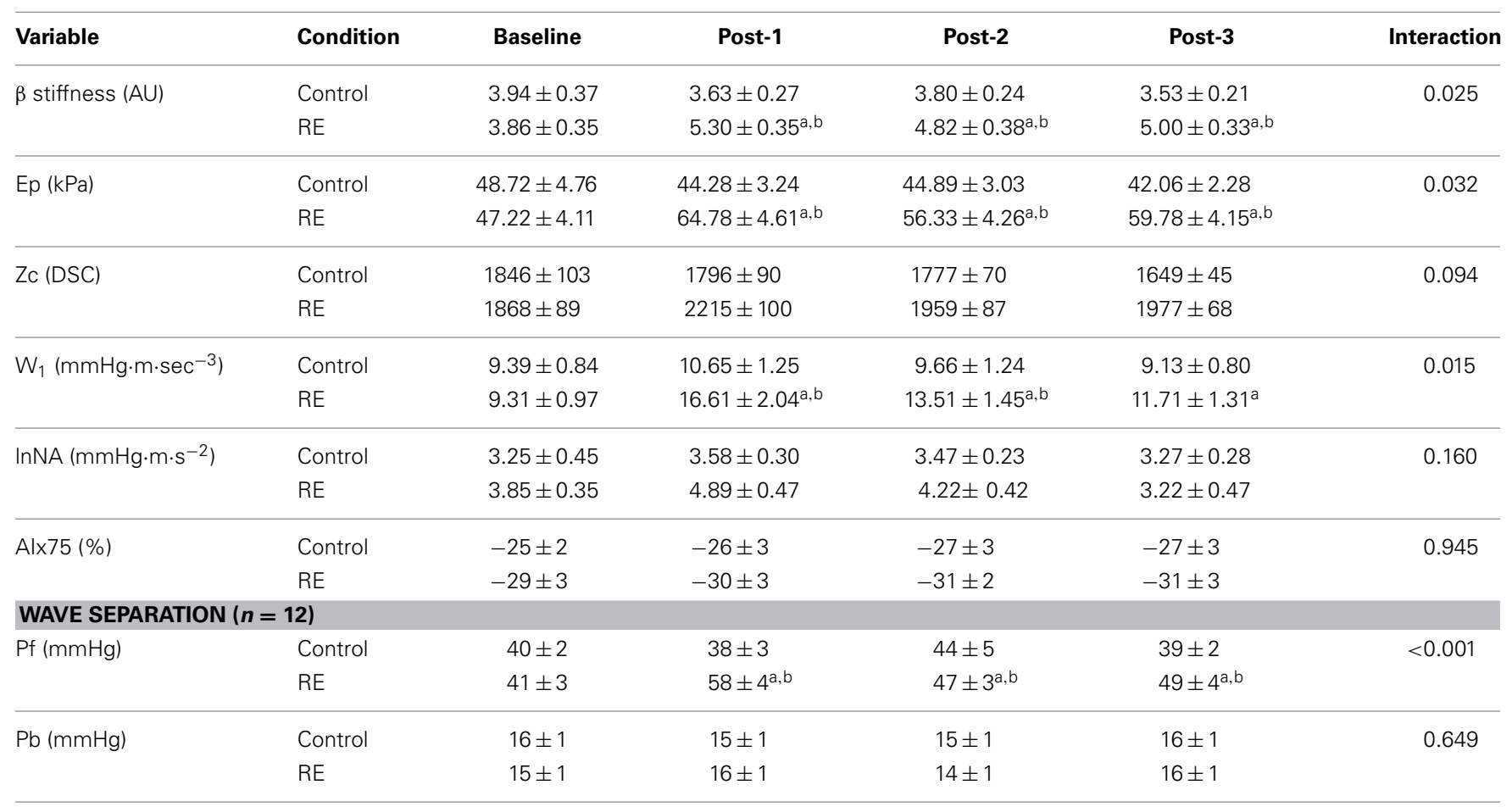

${ }^{a}$ Significantly different from within-condition $B L, p<0.05$.

${ }^{b}$ Significantly different from other condition, same time point, $p<0.05$.

$A / x 75$, augmentation index at a heart rate of $75 \mathrm{bpm}$; NA, negative area; $P f$, forward pressure; $P b$, backwards pressure, DSC, dynes $x \mathrm{~s} / \mathrm{cm}^{5}$.

Table 4 | Measures of aortic wave reflection/stiffness across testing time points between control and resistance exercise $(n=12)$.

\begin{tabular}{|c|c|c|c|c|c|c|}
\hline Variable & Condition & Baseline & Post-1 & Post-2 & Post-3 & Interaction \\
\hline & $\mathrm{RE}$ & $7.1 \pm 0.1$ & $8.1 \pm 0.2$ & $7.6 \pm 0.1$ & $7.3 \pm 0.1$ & \\
\hline \multirow[t]{2}{*}{$\mathrm{Pf}(\mathrm{mmHg})$} & Control & $32 \pm 1$ & $34 \pm 2$ & $34 \pm 2$ & $36 \pm 3$ & 0.867 \\
\hline & $\mathrm{RE}$ & $35 \pm 2$ & $35 \pm 3$ & $38 \pm 3$ & $40 \pm 2$ & \\
\hline \multirow[t]{2}{*}{ Time to $\mathrm{Pb}(\mathrm{ms})$} & Control & $284 \pm 14$ & $276 \pm 11$ & $278 \pm 15$ & $286 \pm 15$ & 0.040 \\
\hline & $\mathrm{RE}$ & $272 \pm 8$ & $249 \pm 5^{a, b}$ & $246 \pm 4^{a, b}$ & $246 \pm 5^{a, b}$ & \\
\hline \multirow[t]{2}{*}{ Alx75 } & Control & $-11 \pm 3$ & $-8 \pm 4$ & $-13 \pm 4$ & $-11 \pm 3$ & 0.013 \\
\hline & $\mathrm{RE}$ & $-9 \pm 2$ & $7 \pm 4$ & $1 \pm 3^{a, b}$ & $-3 \pm 3^{a, b}$ & \\
\hline
\end{tabular}

a significantly different from within-condition $B L, p<0.05$.

${ }^{b}$ significantly different from other condition, same time point, $p<0.05$.

Alx75, augmentation index at a heart rate of 75 bpm; PWV, pulse wave velocity; Pf, forward pressure; $P b$, backwards pressure.

pressure $\left(\mathrm{Pf}\right.$ and $\left.\mathrm{W}_{1}\right)$ and not pressure from wave reflections ( $\mathrm{Pb}$ or $\mathrm{NA})$.

Studies examining cerebrovascular responses to RE have traditionally focused on the steady component of pressure-flow relationships during and immediately after $(<60 \mathrm{~s}) \mathrm{RE}$ and whether changes in mean arterial pressure alter cerebral autoregulation
(Edwards et al., 2002; Pott et al., 2003; Koch et al., 2005). The current study was designed to investigate changes in pulsatile hemodynamics (not the steady component or mean flow) during previously established times of elevated arterial stiffness following RE. The steady and pulsatile components of pressure/flow each differentially associate with cerebral structure and function 
Table 5 | Cerebral variables across testing time points between control and resistance exercise $(n=18)$.

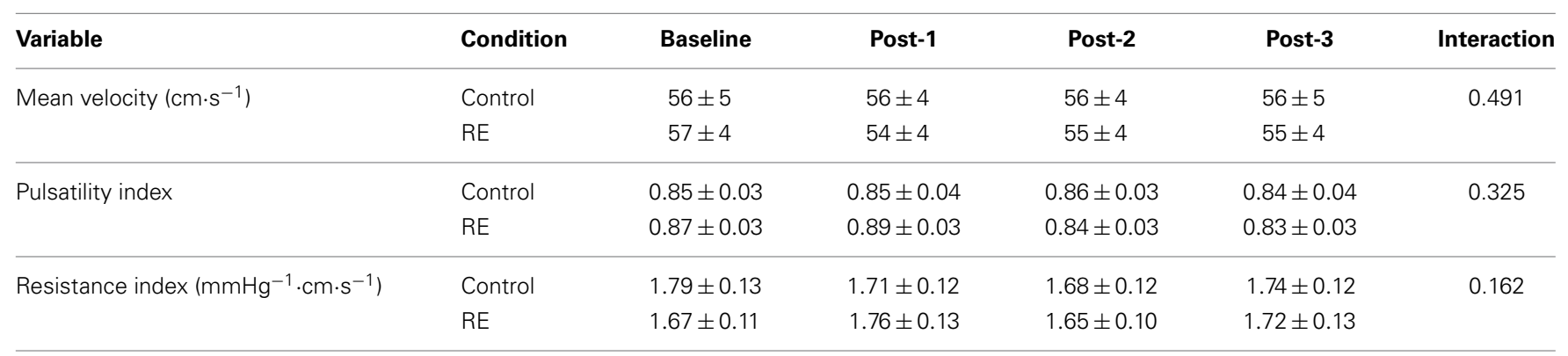

(Mitchell et al., 2011). Many studies note no associations between mean cerebrovascular inflow and cerebral structure/function (Bateman, 2002; Patankar et al., 2006; Henry-Feugeas et al., 2009; Jolly et al., 2013). Conversely, pulsatile flow has been shown to more consistently associate with cerebrovascular damage (Mitchell et al., 2011; Webb et al., 2012; Jolly et al., 2013).

In the present study, despite substantial increases in carotid stiffness for upwards of 30-min after acute RE, there were minimal change in CBFv pulsatility. Carotid artery stiffness increased following RE, reinforcing previous observations regarding the acute effects of RE on central artery stiffness (DeVan et al., 2005; Heffernan et al., 2007b; Fahs et al., 2009; Collier et al., 2010; Yoon et al., 2010). Increased arterial stiffness has previously been linked to pulsatile flow in the cerebrovascular bed (Xu et al., 2012), higher white matter hyperintensities, lower executive function, and increased risk for subcortical infarcts (Mitchell, 2008) separate from the effects of pressure pulsatility. In the current study, RE-induced increases in arterial stiffness did not alter measures of CCA or MCA flow pulsatility. Thus physiological changes in arterial stiffness measured 10-30 min after acute RE may not alter cerebral flow profiles as occurs inherently/chronically with aging or in pathological settings.

Arterial stiffness has also been associated with pulsatile pressure (Tarumi et al., 2011) and in turn impaired cerebrovascular function (Kwater et al., 2009; Webb et al., 2012). In the present study there were increases in carotid pressure pulsatility evidenced by a significant increase in carotid PP post-RE. The blood pressure waveform is an amalgam of forward and backward travelling waves. Left ventricular ejection instigates the genesis of forward travelling pressure waves. These pressure waves may be partially reflected from peripheral vessels with the timing and magnitude of this reflection affected by several hemodynamic factors including arterial stiffness, peripheral vascular tone, and physical distance to the peripheral reflection sites. Using two different novel yet complementary methods of assessing carotid hemodynamics (WIA and WSA), the present study noted that increased pressure pulsatility in the CCA was largely driven by an increase in forward wave pressure (increases in Pf and $\mathrm{W}_{1}$ ) as there were no statistically significant changes in pressure from wave reflections ( $\mathrm{Pb}$ or $\mathrm{NA}$ ). This is consistent with recent findings from Schultz et al. noting that increases in central pressure during exercise are largely mediated by increases in forward wave pressure and not pressure from wave reflections (Schultz et al., 2013). It should be underscored that changes in central hemodynamics following acute RE may not be completely devoid of risk. Clinical consequences of increased forward wave pressure and subsequent pulsatile pressure transmission to the cerebrovascular bed following acute RE require further scrutiny.

Wave reflections detected in the CCA have been shown to be related to altered cerebrovascular tone (Bleasdale et al., 2003; Curtis et al., 2007). Increases in cerebral resistance affect the timing and magnitude of pressure waves being reflected from the head (cerebral circulation) (Bleasdale et al., 2003; Curtis et al., 2007). In general, resistance in the cereobrovascular bed is low allowing for possible transmission of damaging pulsatile energy to penetrate deeply into the intracranial cavity. An increase in cerebrovascular tone would increase pressure from wave reflections (from the brain to the carotid) and might serve to protect against entry of pulsatile energy into the delicate cerebral capillary bed. An increase in wave reflections from the cerebral vasculature would be expected to increase carotid pressure but attenuate flow (Kohara et al., 1999; Curtis et al., 2007). In the current study, there were no statistically significant changes in carotid NA or $\mathrm{Pb}$ (measures of wave reflections) and this mirrored the lack of change seen in carotid and cerebral flow measures. Thus despite large increases in forward wave pressure and overall pressure pulsatility, RE and/or RE-induced stiffness may not alter cerebrovascular flow pulsatility at the time points investigated. In young healthy adults, cerebrovascular flow pulsatility may be dampened via other mechanisms.

Our data offer insight into flow pulsatility buffering following acute RE and suggest that some may occur at the level of the CCA. It has been suggested that as much as a quarter to half of cerebrovascular resistance may be determined at the level of the carotid arteries (Willie et al., 2014). In the present study, the modest increases in CCA Zc after acute RE were associated with reduced CCA PI $(r=-0.61, p<0.05)$. Eighty percent of CCA blood flow at rest feeds the internal carotid artery with subsequent branching giving way to the MCA that in turn supplies approximately $80 \%$ of the blood supply to the brain (Farkas and Luiten, 2001). Compared to the external carotid artery (ECA), the internal carotid artery (ICA) is a lower resistance/impedance vessel with a lower reflection coefficient (Taylor and Tukmachi, 1985) favoring a flow differential at rest. The input impedance of the CCA is thus largely determined by the ECA; parenthetically, the CCA flow pattern approximates that of the lower impedance ICA rather than the ECA (Taylor and Tukmachi, 1985). Owing to the intracranial anastomoses through the orbit, ICA occlusion 


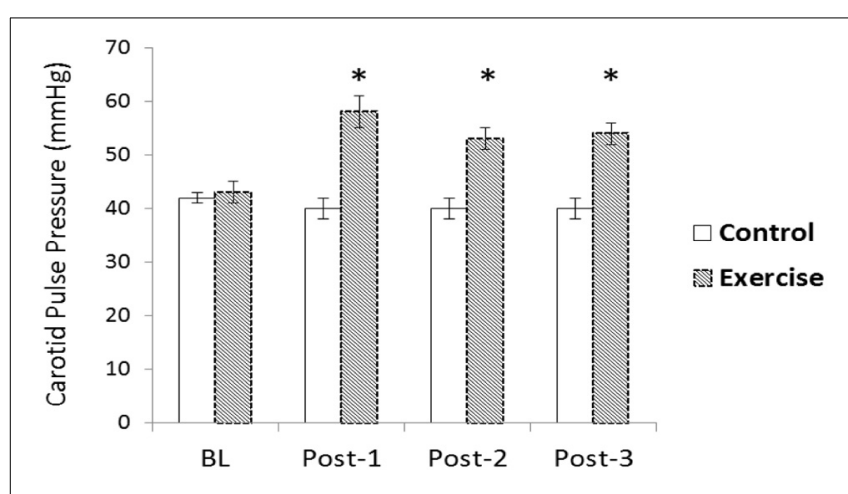

FIGURE 1 | Change in carotid pulse pressure (PP) following acute resistance exercise (RE) vs. a time control condition. A significant condition-by-time interaction was detected for carotid PP $(p<0.05)$. Carotid PP was elevated 10, 20, and 30-min after exercise compared to baseline $(p<0.05)^{*}$

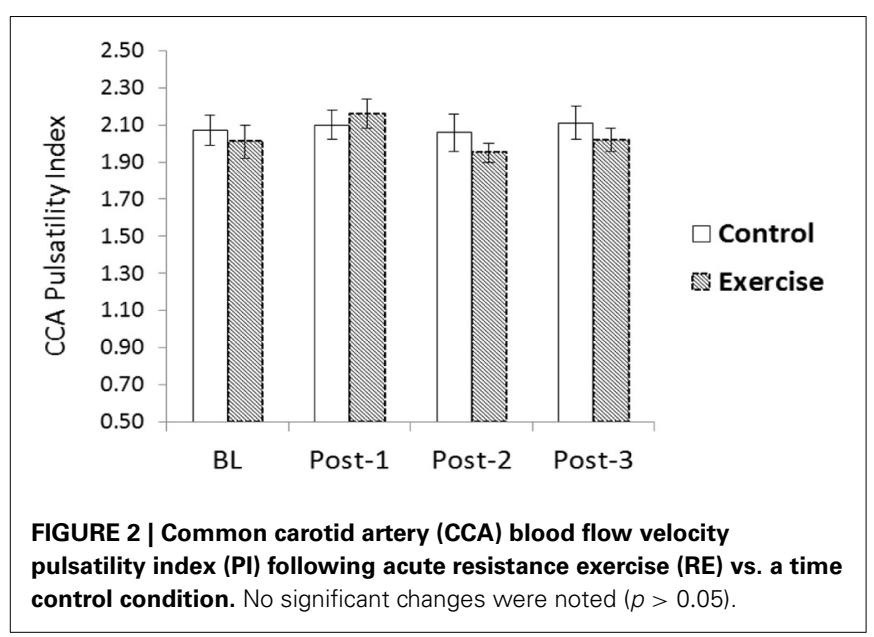

results in flow redistribution to the ipsilateral ECA and a change in the ECA flow profile to match that of a typical ICA/CCA contour (Taylor and Tukmachi, 1985). During exercise, it has been shown that there is an increase in ECA flow relative to the ICA for thermoregulatory purposes (Sato et al., 2011). Taken together and these observations suggest that changes in CCA/ICA/ECA impedance matching during/following exercise may affect pulsatile flow transmission at these crucial interfaces.

Some buffering of hemodynamic pulsatility may also occur at the carotid-aortic interface (Mitchell et al., 2011). We noted disparate changes in carotid and aortic hemodynamics following acute RE. While there was no change in carotid AIx, there was a significant increase in aortic AIx following acute RE (Yoon et al., 2010). Increases in aortic AIx were due to changes in timing of wave reflection travel likely from an increase in aortic PWV and not changes in magnitude as there were minimal changes in aortic $\mathrm{Pb}$. Arterial stiffness and pressure from wave reflections have been shown to affect retrograde flow in the femoral/aorta (Hashimoto and Ito, 2010; Heffernan et al., 2013) which has been linked to flow in the CCA (Hashimoto and Ito, 2013). There were also disparate changes in forward wave pressure (Pf) between
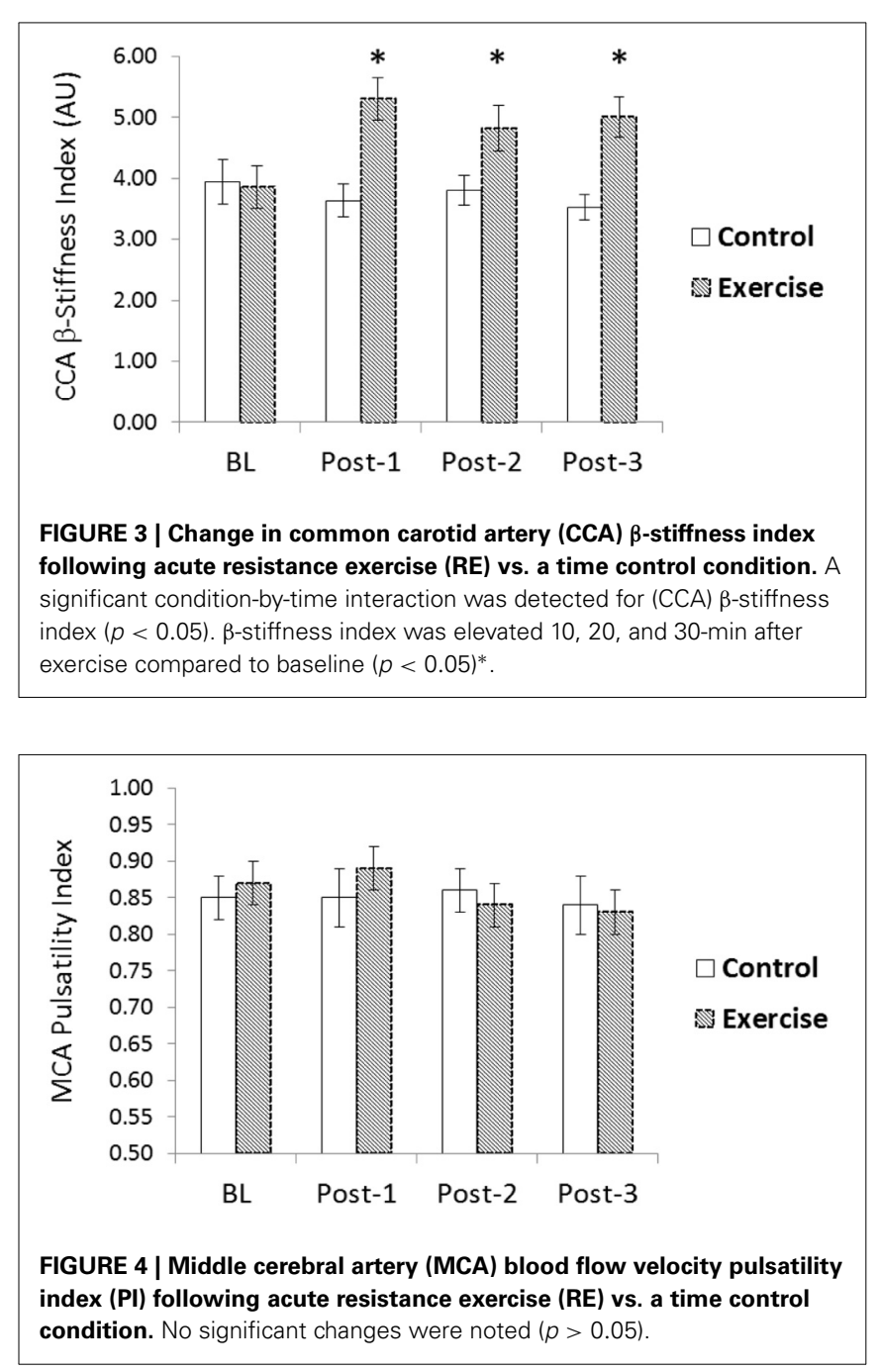

CCA and aorta. Thus, alterations in impedance matching at the carotid-aorta interface may also affect CCA pressure and flow pulsaility following acute RE. An additional alternative mechanism for buffering of flow pulsatility to the MCA may reside in the geometry of the ICA. The carotid siphon describes the tortuous distal part of the internal carotid artery that may bend beyond $180^{\circ}$ with minimal inter-individual variability (Schubert et al., 2011). This shape has been shown to enhance pulsatile energy dispersion and markedly attenuate flow pulsatility in vivo (Schubert et al., 2011). Finally, it is also possible that some hemodynamic buffering occurs within the intracranial vessels via their capacitance properties. The cerebral Windkessel may extinguish pulsatilite hemodynamic energy via matching cerebrospinal fluid ejection to venous flow volume out of the intracranial cavity (Chan et al., 2011).

Elevations in HR after acute RE support previous studies noting increases in sympathetic nervous system (SNS) activity during recovery from this exercise modality (Heffernan et al., 2006). The role of the SNS in modulating cerebral hemodynamics in humans remains conflicting (Willie et al., 2014). While select studies suggest an important role for the SNS in affecting mean flow 
patterns and autoregulation in cerebral vessels, studies directly examining associations between the SNS and cerebrovascular flow pulsatility are lacking. The effect of SNS activity on carotid artery mechanical properties in humans are also conflicting with some studies suggesting a link (Sugawara et al., 2009a; Liu et al., 2011) and others noting no association (Kosch et al., 2002). It is possible that increases in sympathetic activation following acute RE contribute to increases in CCA stiffness/impedance while concomitantly preventing forced dilation of the cerebral arterioles; both of which may prevent regional over-perfusion and protect against blood-brain barrier breakdown from transmission of excessive pressure/flow fluctuations (Ogoh and Ainslie, 2009).

MCA PI is elevated immediately (within 2-min) after acute RE (Koch et al., 2005) thus it is possible an important window for data acquisition was missed. We intentionally chose not to assess vascular properties during this time point as marked changes in MAP, HR and ventilation with subsequent changes in $\mathrm{CO}_{2}$ could confound interpretation of findings (Wilkinson et al., 2002; Romero and Cooke, 2007). Of central importance for the present study and in accordance with previously published studies (DeVan et al., 2005) CCA stiffness was elevated at all time-points assessed. Despite these changes in CCA stiffness, there was no change in CCA or MCA flow pulsatility at these time points reinforcing our conclusion that acute RE-mediated increases in arterial stiffness may not detrimentally impact cerebrovascular flow pulsatility. Future research is needed to explore central hemodynamic and cerebrovascular changes during earlier time points following acute RE.

\section{LIMITATIONS}

Additional limitations to this study should be noted. This study utilized healthy young men. Results may not be directly applicable to women, older adults or other clinical populations. TCD cannot measure blood flow per se since diameter is not measured. Although previous research has suggested that the diameter of large cerebral vessels do not change across different physiological stimuli (Stroobant and Vingerhoets, 2000), this has never been demonstrated empirically following acute exercise. The WSA method used in this study was not completed on 6 participants due to wave reflection times occurring outside of the analysis software's acceptable range (reflection times $<50 \mathrm{~ms}$ ). This occurred most notably after acute RE. It is possible that RE-induced tachycardia and the concomitant decrease in ejection duration changed wave reflection timing such that reflected waves arrived during early systole. Despite incomplete results using this technique, values obtained from the subset of participants with complete CCA WSA data confirmed findings from CCA WIA. Finally, it must be noted that cerebral autoregulation was not measured as it was not the purpose of this investigation. Cerebrovascular regulation is preserved following acute aerobic exercise (Willie et al., 2013). Future research is needed to explore associations between CCA stiffness and cerebral autoregulation during/following various physiologic stressors including resistance exercise.

\section{CONCLUSION}

Acute RE increases carotid artery stiffness and pressure pulsatility without affecting cerebral flow pulsatility. RE-induced increases in carotid pressure pulsatility may be due to increases in forward wave pressure and not pressure from wave reflections.

\section{ACKNOWLEDGMENTS}

No external funding was provided for this study. We thank all participants for the generous giving of their time and arteries.

\section{REFERENCES}

Bateman, G. A. (2002). Pulse-wave encephalopathy: a comparative study of the hydrodynamics of leukoaraiosis and normal-pressure hydrocephalus. Neuroradiology 44, 740-748. doi: 10.1007/s00234-002-0812-0

Bleasdale, R. A., Mumford, C. E., Campbell, R. I., Fraser, A. G., Jones, C. J., and Frenneaux, M. P. (2003). Wave intensity analysis from the common carotid artery: a new noninvasive index of cerebral vasomotor tone. Heart Vessels 18, 202-206. doi: 10.1007/s00380-003-0711-2

Carallo, C., Irace, C., Pujia, A., De Franceschi, M. S., Crescenzo, A., Motti, C., et al. (1999). Evaluation of common carotid hemodynamic forces. Relations with wall thickening. Hypertension 34, 217-221. doi: 10.1161/01.HYP.34.2.217

Chan, G. S., Ainslie, P. N., Willie, C. K., Taylor, C. E., Atkinson, G., Jones, H., et al. (2011). Contribution of arterial Windkessel in low-frequency cerebral hemodynamics during transient changes in blood pressure. J. Appl. Physiol. (1985) 110, 917-925. doi: 10.1152/japplphysiol.01407.2010

Collier, S. R., Diggle, M. D., Heffernan, K. S., Kelly, E. E., Tobin, M. M., and Fernhall, B. (2010). Changes in arterial distensibility and flow-mediated dilation after acute resistance vs. aerobic exercise. J. Strength Cond. Res. 24, 2846-2852. doi: 10.1519/JSC.0b013e3181e840e 0

Curtis, S. L., Zambanini, A., Mayet, J., McG Thom, S. A., Foale, R., Parker, K. H., et al. (2007). Reduced systolic wave generation and increased peripheral wave reflection in chronic heart failure. Am. J. Physiol. Heart Circ. Physiol. 293, H557-H562. doi: 10.1152/ajpheart.01095.2006

DeVan, A. E., Anton, M. M., Cook, J. N., Neidre, D. B., Cortez-Cooper, M. Y., and Tanaka, H. (2005). Acute effects of resistance exercise on arterial compliance. J. Appl. Physiol. 98, 2287-2291. doi: 10.1152/japplphysiol.00002.2005

Edwards, M. R., Martin, D. H., and Hughson, R. L. (2002). Cerebral hemodynamics and resistance exercise. Med. Sci. Sports Exerc. 34, 1207-1211. doi: 10.1097/00005768-200207000-00024

Fahs, C. A., Heffernan, K. S., and Fernhall, B. (2009). Hemodynamic and vascular response to resistance exercise with L-arginine. Med. Sci. Sports Exerc. 41, 773-779. doi: 10.1249/MSS.0b013e3181909d9d

Farkas, E., and Luiten, P. G. (2001). Cerebral microvascular pathology in aging and Alzheimer's disease. Prog. Neurobiol. 64, 575-611. doi: 10.1016/S03010082(00)00068-X

Filipovsky, J., Mayer, O., Dolejsova, M., and Seidlerova, J. (2010). The assessment of carotid-femoral distance for aortic pulse wave velocity: should it be estimated from body height? Artery Res. 4, 19-23. doi: 10.1016/j.artres.2010.01.002

Hanya, S. (2013). Validity of the water hammer formula for determining regional aortic pulse wave velocity: comparison of one-point and two-point (Foot-toFoot) measurements using a multisensor catheter in human. Ann. Vasc. Dis. 6, 150-158. doi: 10.3400/avd.oa.13-00046

Harada, A., Okada, T., Niki, K., Chang, D., and Sugawara, M. (2002). On-line noninvasive one-point measurements of pulse wave velocity. Heart Vessels 17, 61-68. doi: $10.1007 / \mathrm{s} 003800200045$

Hashimoto, J., and Ito, S. (2010). Pulse pressure amplification, arterial stiffness, and peripheral wave reflection determine pulsatile flow waveform of the femoral artery. Hypertension 56, 926-933. doi: 10.1161/HYPERTENSIONAHA.110.159368

Hashimoto, J., and Ito, S. (2013). Aortic stiffness determines diastolic blood flow reversal in the descending thoracic aorta: potential implication for retrograde embolic stroke in hypertension. Hypertension 62, 542-549. doi: 10.1161/HYPERTENSIONAHA.113.01318

Heffernan, K. S., Collier, S. R., Kelly, E. E., Jae, S. Y., and Fernhall, B. (2007a). Arterial stiffness and baroreflex sensitivity following bouts of aerobic and resistance exercise. Int. J. Sports Med. 28, 197-203. doi: 10.1055/s-2006924290

Heffernan, K. S., Jae, S. Y., Echols, G. H., Lepine, N. R., and Fernhall, B. (2007b) Arterial stiffness and wave reflection following exercise in resistance-trained men. Med. Sci. Sports Exerc. 39, 842-848. doi: 10.1249/mss.0b013e318031b03c 
Heffernan, K. S., Kelly, E. E., Collier, S. R., and Fernhall, B. (2006). Cardiac autonomic modulation during recovery from acute endurance vs. resistance exercise. Eur. J. Cardiovasc. Prev. Rehabil. 13, 80-86. doi: 10.1097/01.hjr.0000197470.74070.46

Heffernan, K. S., Lefferts, W. K., Kasprowicz, A. G., Tarzia, B. J., Thijssen, D. H., and Brutsaert, T. D. (2013). Manipulation of arterial stiffness, wave reflections, and retrograde shear rate in the femoral artery using lower limb external compression. Physiol. Rep. 1, e00022. doi: 10.1002/phy2.22

Henry-Feugeas, M. C., Roy, C., Baron, G., and Schouman-Claeys, E. (2009). Leukoaraiosis and pulse-wave encephalopathy: observations with phasecontrast MRI in mild cognitive impairment. J. Neuroradiol. 36, 212-218. doi: 10.1016/j.neurad.2009.01.003

Jolly, T. A., Bateman, G. A., Levi, C. R., Parsons, M. W., Michie, P. T., and Karayanidis, F. (2013). Early detection of microstructural white matter changes associated with arterial pulsatility. Front. Hum. Neurosci. 7:782. doi: 10.3389/fnhum. 2013.00782

Jung, J., Kang, H., Shim, S., Cho, K., and Yu, J. (2012). Effects of resistive exercise on cerebral blood flow velocity and pulsatility index of healthy people. J. Phys. Ther. Sci. 24, 915-917. doi: 10.1589/jpts.24.915

Katsi, V., Vlachopoulos, C., Souretis, G., Baou, K., Dagalaki, I., Alexopoulos, N., et al. (2012). Association between retinal microcirculation and aortic stiffness in hypertensive patients. Int. J. Cardiol. 157, 370-373. doi: 10.1016/j.ijcard.2010.12.074

Koch, A., Ivers, M., Gehrt, A., Schnoor, P., Rump, A., and Rieckert, H. (2005). Cerebral autoregulation is temporarily disturbed in the early recovery phase after dynamic resistance exercise. Clin. Auton. Res. 15, 83-91. doi: $10.1007 /$ s10286-005-0249-8

Kohara, K., Jiang, Y., Igase, M., and Hiwada, K. (1999). Effect of reflection of arterial pressure on carotid circulation in essential hypertension. Am. J. Hypertens. 12, 1015-1020. doi: 10.1016/S0895-7061(99)00091-6

Kosch, M., Barenbrock, M., Kisters, K., Rahn, K. H., and Hausberg, M. (2002). Relationship between muscle sympathetic nerve activity and large artery mechanical vessel wall properties in renal transplant patients. J. Hypertens. 20, 501-508. doi: 10.1097/00004872-200203000-00026

Kwater, A., Gasowski, J., Gryglewska, B., Wizner, B., and Grodzicki, T. (2009). Is blood flow in the middle cerebral artery determined by systemic arterial stiffness? Blood Press. 18, 130-134. doi: 10.1080/08037050902 975114

Liu, J., Cao, T. S., Duan, Y. Y., Yang, Y. L., and Yuan, L. J. (2011). Effects of cold pressor-induced sympathetic stimulation on the mechanical properties of common carotid and femoral arteries in healthy males. Heart Vessels 26, 214-221. doi: 10.1007/s00380-010-0069-1

Mitchell, G. F. (2008). Effects of central arterial aging on the structure and function of the peripheral vasculature: implications for end-organ damage. J. Appl. Physiol. 105, 1652-1660. doi: 10.1152/japplphysiol.90549.2008

Mitchell, G. F., Hwang, S. J., Vasan, R. S., Larson, M. G., Pencina, M J., Hamburg, N. M., et al. (2010). Arterial stiffness and cardiovascular events: the Framingham Heart Study. Circulation 121, 505-511. doi: 10.1161/CIRCULATIONAHA.109.886655

Mitchell, G. F., van Buchem, M. A., Sigurdsson, S., Gotal, J. D., Jonsdottir, M. K., Kjartansson, O., et al. (2011). Arterial stiffness, pressure and flow pulsatility and brain structure and function: the Age, Gene/Environment Susceptibility-Reykjavik study. Brain 134, 3398-3407. doi: 10.1093/brain/ awr253

Mitchell, G. F., Vita, J. A., Larson, M. G., Parise, H., Keyes, M. J., Warner, E., et al. (2005). Cross-sectional relations of peripheral microvascular function, cardiovascular disease risk factors, and aortic stiffness: the Framingham Heart Study. Circulation 112, 3722-3728. doi: 10.1161/CIRCULATIONAHA.105. 551168

Miyachi, M. (2013). Effects of resistance training on arterial stiffness: a metaanalysis. Br. J. Sports Med. 47, 393-396. doi: 10.1136/bjsports-2012-090488

Miyachi, M., Kawano, H., Sugawara, J., Takahashi, K., Hayashi, K., Yamazaki, K., et al. (2004). Unfavorable effects of resistance training on central arterial compliance: a randomized intervention study. Circulation 110, 2858-2863. doi: 10.1161/01.CIR.0000146380.08401.99

Niki, K., Sugawara, M., Chang, D., Harada, A., Okada, T., Sakai, R., et al. (2002). A new noninvasive measurement system for wave intensity: evaluation of carotid arterial wave intensity and reproducibility. Heart Vessels 17, 12-21. doi: $10.1007 / \mathrm{s} 003800200037$
Ogoh, S., and Ainslie, P. N. (2009). Cerebral blood flow during exercise: mechanisms of regulation. J. Appl. Physiol. (1985) 107, 1370-1380. doi: 10.1152/japplphysiol.00573.2009

Patankar, T., Widjaja, E., Chant, H., McCollum, C., Baldwin, R., Jeffries, S., et al. (2006). Relationship of deep white matter hyperintensities and cerebral blood flow in severe carotid artery stenosis. Eur. J. Neurol. 130, 10-16. doi: 10.1111/j.1468-1331.2006.01115.x

Pollock, M. L., Franklin, B. A., Balady, G. J., Chaitman, B. L., Fleg, J. L., Fletcher, B., et al. (2000). AHA Science Advisory. Resistance exercise in individuals with and without cardiovascular disease: benefits, rationale, safety, and prescription: an advisory from the committee on exercise, rehabilitation, and prevention, council on clinical cardiology, american heart association; position paper endorsed by the american college of sports medicine. Circulation 101, 828-833. doi: 10.1161/01.CIR.101.7.828

Pott, F., Van Lieshout, J. J., Ide, K., Madsen, P., and Secher, N. H. (2003). Middle cerebral artery blood velocity during intense static exercise is dominated by a Valsalva maneuver. J. Appl. Physiol. 94, 1335-1344. doi: 10.1152/japplphysiol.00457.2002

Qasem, A., and Avolio, A. (2008). Determination of aortic pulse wave velocity from waveform decomposition of the central aortic pressure pulse. Hypertension 51 , 188-195. doi: 10.1161/HYPERTENSIONAHA.107.092676

Romero, S. A., and Cooke, W. H. (2007). Hyperventilation before resistance exercise: cerebral hemodynamics and orthostasis. Med. Sci. Sports Exerc. 39, 1302-1307. doi: 10.1249/mss.0b013e3180653636

Safar, M. E., Nilsson, P. M., Blacher, J., and Mimran, A. (2012). Pulse pressure, arterial stiffness, and end-organ damage. Curr. Hypertens. Rep. 14, 339-344. doi: $10.1007 /$ s11906-012-0272-9

Sato, K., Ogoh, S., Hirasawa, A., Oue, A., and Sadamoto, T. (2011). The distribution of blood flow in the carotid and vertebral arteries during dynamic exercise in humans. J. Physiol. 589, 2847-2856. doi: 10.1113/jphysiol.2010.204461

Schubert, T., Santini, F., Stalder, A. F., Bock, J., Meckel, S., Bonati, L., et al. (2011). Dampening of blood-flow pulsatility along the carotid siphon: does form follow function? AJNR Am. J. Neuroradiol. 32, 1107-1112. doi: 10.3174/ ajnr.A2426

Schultz, M. G., Davies, J. E., Roberts-Thomson, P., Black, J. A., Hughes, A. D., and Sharman, J. E. (2013). Exercise central (aortic) blood pressure is predominantly driven by forward traveling waves, not wave reflection. Hypertension 62, 175-182. doi: 10.1161/HYPERTENSIONAHA.111.00584

Stroobant, N., and Vingerhoets, G. (2000). Transcranial Doppler ultrasonography monitoring of cerebral hemodynamics during performance of cognitive tasks: a review. Neuropsychol. Rev. 10, 213-231. doi: 10.1023/A:1026412811036

Sugawara, J., Komine, H., Hayashi, K., Yoshizawa, M., Otsuki, T., Shimojo, N., et al. (2009a). Reduction in alpha-adrenergic receptor-mediated vascular tone contributes to improved arterial compliance with endurance training. Int. J. Cardiol. 135, 346-352. doi: 10.1016/j.ijcard.2008.04.007

Sugawara, M., Niki, K., Ohte, N., Okada, T., and Harada, A. (2009b). Clinical usefulness of wave intensity analysis. Med. Biol. Eng. Comput. 47, 197-206. doi: 10.1007/s11517-008-0388-x

Sutton-Tyrrell, K., Najjar, S. S., Boudreau, R. M., Venkitachalam, L., Kupelian, V., Simonsick, E. M., et al. (2005). Elevated aortic pulse wave velocity, a marker of arterial stiffness, predicts cardiovascular events in well-functioning older adults. Circulation 111, 3384-3390. doi: 10.1161/CIRCULATIONAHA.104. 483628

Tarumi, T., Shah, F., Tanaka, H., and Haley, A. P. (2011). Association between central elastic artery stiffness and cerebral perfusion in deep subcortical gray and white matter. Am. J. Hypertens. 24, 1108-1113. doi: 10.1038/ajh.2011.101

Taylor, D. E., and Tukmachi, E. S. (1985). Contribution of internal and external carotid beds to common carotid artery input impedance in the dog. Q. J. Exp. Physiol. 70, 177-187.

Van Bortel, L. M., Balkestein, E. J., van der Heijden-Spek, J. J., Vanmolkot, F. H., Staessen, J. A., Kragten, J. A., et al. (2001). Non-invasive assessment of local arterial pulse pressure: comparison of applanation tonometry and echo-tracking. J. Hypertens. 19, 1037-1044. doi: 10.1097/00004872-200106000-00007

Webb, A. J., Simoni, M., Mazzucco, S., Kuker, W., Schulz, U., and Rothwell, P. M. (2012). Increased cerebral arterial pulsatility in patients with leukoaraiosis: arterial stiffness enhances transmission of aortic pulsatility. Stroke 43, 2631-2636. doi: 10.1161/STROKEAHA.112.655837

Westerhof, B. E., Guelen, I., Westerhof, N., Karemaker, J. M., and Avolio, A. (2006). Quantification of wave reflection in the human aorta from 
pressure alone: a proof of principle. Hypertension 48, 595-601. doi: 10.1161/01.HYP.0000238330.08894.17

Wilkinson, I. B., Mohammad, N. H., Tyrrell, S., Hall, I. R., Webb, D. J., Paul, V. E., et al. (2002). Heart rate dependency of pulse pressure amplification and arterial stiffness. Am. J. Hypertens. 15, 24-30. doi: 10.1016/S0895-7061(01)02252-X

Willie, C. K., Ainslie, P. N., Taylor, C. E., Eves, N. D., and Tzeng, Y. C. (2013). Maintained cerebrovascular function during post-exercise hypotension. Eur. J. Appl. Physiol. 113, 1597-1604. doi: 10.1007/s00421-012-2578-3

Willie, C. K., Tzeng, Y. C., Fisher, J. A., and Ainslie, P. N. (2014). Integrative regulation of human brain blood flow. J. Physiol. 592, 841-859. doi: 10.1113/jphysiol. 2013.268953

Xu, T. Y., Staessen, J. A., Wei, F. F., Xu, J., Li, F. H., Fan, W. X., et al. (2012). Blood flow pattern in the middle cerebral artery in relation to indices of arterial stiffness in the systemic circulation. Am. J. Hypertens. 25, 319-324. doi: 10.1038/ajh.2011.223

Yoon, E. S., Jung, S. J., Cheun, S. K., Oh, Y. S., Kim, S. H., and Jae, S. Y. (2010). Effects of acute resistance exercise on arterial stiffness in young men. Korean Circ. J. 40, 16-22. doi: 10.4070/kcj.2010.40.1.16
Conflict of Interest Statement: The authors declare that the research was conducted in the absence of any commercial or financial relationships that could be construed as a potential conflict of interest.

Received: 02 January 2014; paper pending published: 23 January 2014; accepted: 28 February 2014; published online: 19 March 2014.

Citation: Lefferts WK, Augustine JA and Heffernan KS (2014) Effect of acute resistance exercise on carotid artery stiffness and cerebral blood flow pulsatility. Front. Physiol. 5:101. doi: 10.3389/fphys.2014.00101

This article was submitted to Integrative Physiology, a section of the journal Frontiers in Physiology.

Copyright (c) 2014 Lefferts, Augustine and Heffernan. This is an open-access article distributed under the terms of the Creative Commons Attribution License (CC BY). The use, distribution or reproduction in other forums is permitted, provided the original author(s) or licensor are credited and that the original publication in this journal is cited, in accordance with accepted academic practice. No use, distribution or reproduction is permitted which does not comply with these terms. 